See discussions, stats, and author profiles for this publication at: https://www.researchgate.net/publication/285236808

\title{
Hidden High Period Accelerator Modes in a Bouncer Model
}

Chapter $\cdot$ November 2015

DOI: 10.1007/978-3-319-24871-4_13

\section{CITATIONS}

0

4 authors:

(2) Tiago Kroetz

Universidade Tecnológica Federal do Paraná - Pato Branco 36 PUBLICATIONS 145 CITATIONS

SEE PROFILE

Edson D. Leonel

São Paulo State University

154 PUblications 1,396 CITATIONS

SEE PROFILE
André L P Livorati

São Paulo State University

28 PUBLICATIONS 186 CITATIONS

SEE PROFILE

Ibere Luiz Caldas

University of São Paulo

349 PUBLICATIONS 2,570 CITATIONS

SEE PROFILE

Some of the authors of this publication are also working on these related projects:

Synchronization of Neurons View project

Chaotic motion View project 


\title{
Hidden High Period Accelerator Modes in a Bouncer Model
}

\author{
Tiago Kroetz, André L.P. Livorati, Edson D. Leonel \\ and Iberê L. Caldas
}

\begin{abstract}
We characterized the influence of high period accelerator modes in the global dynamics of a non-dissipative Bouncer model. The dynamics of the system was investigated considering both complete and simplified approaches. Evaluating the average of the velocity over large ensembles of initial conditions for the complete mapping, we obtained particular ranges of the control parameter where high period accelerating structures are located. The position, influence and shape of the accelerator modes were obtained considering the symplectic mapping. Our results, lead us to infer that even for high period and less influent accelerator modes, the dynamics is globally affected for long time series, causing an anomalous diffusion, in compare with the regular Fermi acceleration.
\end{abstract}

\section{Introduction}

Modelling of dynamical systems, has been one of the most embracing area of interest among physicists and mathematicians is the past decades [1-5]. Low-dimensional systems in particular, despite the simple modelling, are very suitable to study and to investigate chaotic properties in their phase space [1-5]. These systems can present a very complex dynamics leading to a rich variety of nonlinear phenomena, considering either dissipative and non-dissipative dynamics [1-5].

T. Kroetz ( $\varangle)$

Universidade Tecnológica Federal do Paraná, Pato Branco, Paraná, Brazil

e-mail: kroetzfisica@gmail.com

\author{
A.L.P. Livorati · E.D. Leonel \\ Departamento de Física, Universidade Estadual Paulista, Rio Claro,São Paulo, Brazil \\ e-mail: livorati@rc.unesp.br \\ E.D. Leonel \\ e-mail: edleonel@rc.unesp.br \\ I.L. Caldas \\ Instituto de Física, Universidade de São Paulo, São Paulo, Brazil \\ e-mail: ibere@if.usp.br


The Italian physicist Enrico Fermi [6] proposed in 1949, a mechanism as an attempt to explain the origin of the high energies of the cosmic rays. Fermi claimed that charged particles, which interacted with oscillating magnetic fields present in the cosmos, would in the average exhibit a gain of energy. This unlimited growth of energy is denominated Fermi acceleration (FA) and has many applications in several areas of research, as Plasma Physics [7, 8], Astrophysics [9, 10], Atomoptics [11, 12], and specially in billiard dynamics [13-18]. This unlimited energy growth is mainly associated with normal diffusion in phase space. However, FA may present distinct transport from the normal diffusion, as exponential [19-22], or where stickiness phenomenon [4, 5] plays the role of a slowing mechanism for FA [22].

The Bouncer Model [23-25] will be the focus of our study in this paper, where basically we have a free particle under the influence of a constant gravitational field suffering elastic collisions with a vibrating platform. In the non-dissipative version and depending on both control parameters and initial conditions, the bouncer ball presents FA [1]. Despite the simple dynamics, applications for this model can be found in dynamic stability in human performance, [26], vibrations waves in a nanometric-sized mechanical contact system [27], mechanical vibrations [28, 29], anomalous transport and diffusion [30], thermodynamics [31], chaos control [32, 33], granular materials [34-36], among others.

In this paper we investigate how resonances in the phase space, known as accelerator modes (AM) (or ballistic modes) [37-48], influences diffusion and transport properties of the average velocity of the Bouncer Model. We observed that in the presence of this accelerating structures, the dynamics behaves in a regular and monotonic increase of velocity, differing from the normal diffusion present by the "regular FA" $[22,49]$. By exploring the the non-symplectic character of the mapping, we set a numerical search for the AM of high period and low influence on the global dynamics [49]. We found a series of hidden AM, which accelerate more the dynamics for long times than normal diffusion, where their shape and evolution were characterized by stability islands in the symplectic version of the map. We observed that for sufficient long time series, the presence of any AM, of high period or not, would affect globally the dynamics of the system.

The paper is organized as follows: In Sect. 2, we describe the dynamics of the Bouncer Model, in both symplectic and non-symplectic version. Section 3 is devoted to determine analytically the existence of period-1 accelerator mode. Also, we perform a numerical search for high period AM and characterized their period and control parameter range of stability in a table. In Sect. 4 we use the symplectic version of the model to reveal the accelerating structures in the the modulated phase space. Finally, in Sect. 5 we draw some final remarks and conclusions.

\section{The Model}

The dynamical system used to explore the existence of AM with high period and low influence is the Bouncer Model. The dynamical analysis of this system can be explored considering two versions of a two dimensional mapping. The complete 
Fig. 1 Schematic view of Bouncer model

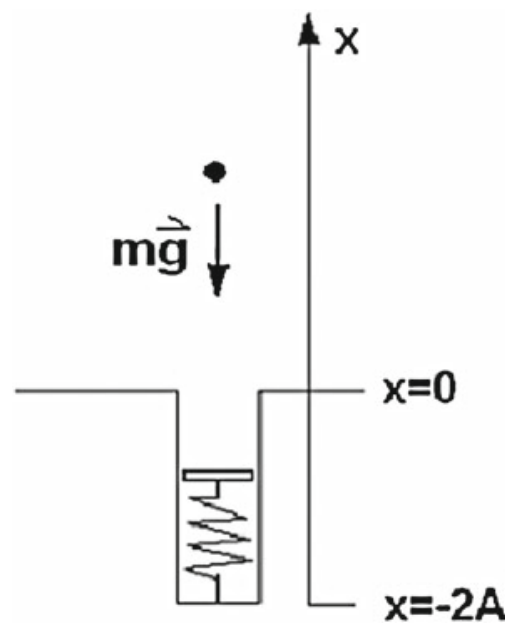

one (non-symplectic) consists in considering the full dynamics of a bouncing ball colliding elastically with a vibrating platform. The simplified version (symplectic), is set by considering the position of the platform fixed. However, when occurs a colision between the particle and the platform, they exchange momentum and energy as if the oscillatory platform where normally moving. A schematic view of the Bouncer Model is shown in Fig. 1.

In the complete version, the position of the oscillatory platform is given by $x_{w}(t)=$ $A[\cos (\omega t+\varphi)-1]$, where $A$ is the amplitude of the platform oscillation, $\omega$ is the angular frequency and $\varphi$ is the oscillation initial phase. The gravitational field acts as a return mechanism which causes repeated impacts between the particle and the platform.

The position of the particle between impacts is given by the free fall equation $x_{p}(t)=h_{0}+v t-g t^{2} / 2$, where $h_{0}$ is the vertical position from which the particle was previously launched by the platform, $v$ is the launch velocity, $t$ is the time elapsed since the last impact and $g$ is the gravitational acceleration. The instants of impacts are obtained by equating the platform position and the particle position $x_{w}(t)=x_{p}(t)$.

\subsection{Complete Model}

The velocity of the particle after each impact will be given by the negative relative velocity between the particle and the platform just before the collision. Obtaining the velocity of the platform and of the particle in a recurrent way at each impact, we can write a set of discrete equations representing the particle velocity $v_{n}$ and of the phase of the platform movement $\varphi_{n}$. The time interval $t_{n+1}$ and the phase difference 
between two consecutive impacts are related by $t_{n+1}=\left(\varphi_{n+1}-\varphi_{n}\right) / \omega$. Therefore, the discrete map that describes the complete Bouncer Model can be written as

$$
\left\{\begin{array}{l}
A\left[\cos \left(\varphi_{n}\right)-\cos \left(\varphi_{n+1}\right)\right]+v_{n}\left(\varphi_{n+1}-\varphi_{n}\right) / \omega-g\left(\varphi_{n+1}-\varphi_{n}\right)^{2} / 2 \omega^{2}=0 \\
v_{n+1}=-v_{n}+g\left(\varphi_{n+1}-\varphi_{n}\right) / \omega-2 A \omega \sin \left(\varphi_{n+1}\right)
\end{array} .\right.
$$

The first expression in (1) must be solved numerically at each collision in order to find the value of $\varphi_{n+1}$.

Defining a parameter $K$ in terms of $A, \omega$ and $g$ as $K=\omega^{2} A /(\pi g)$ it is possible to write a new map dependent on a single control parameter. This parameter is interpreted as a ratio between accelerations of the moving platform and the gravitational field. To perform this procedure, we rewrite the map in terms of a dimensionless velocity given by $V_{n}=\omega v_{n} /(\pi g)$. So, we write the new map as

$$
\left\{\begin{array}{l}
K\left[\cos \left(\varphi_{n}\right)-\cos \left(\varphi_{n+1}\right)\right]+V_{n}\left(\varphi_{n+1}-\varphi_{n}\right)\left(\varphi_{n+1}-\varphi_{n}\right)^{2} / 2 \pi=0 \\
V_{n+1}=-V_{n}+\left(\varphi_{n+1}-\varphi_{n}\right) / \pi-2 K \sin \left(\varphi_{n+1}\right)
\end{array} .\right.
$$

To determine the non-symplectic character of this model, we need to obtain the determinant of Jacobian matrix associated with the mapping expressed in (2). The volume element in phase space varies according this determinant, whose elements are given by the partial derivatives of the mapping variables. The partial derivatives of $\varphi_{n+1}$ are obtained by indirect differentiation of the first expression of the mapping (2), followed by some algebra in order to isolate the terms. These expressions are written as

$$
\begin{gathered}
\frac{\partial \varphi_{n+1}}{\partial \varphi_{n}}=\frac{V_{n}-\left(\varphi_{n+1}-\varphi_{n}\right) / \pi+K \sin \left(\varphi_{n}\right)}{V_{n}-\left(\varphi_{n+1}-\varphi_{n}\right) / \pi+K \sin \left(\varphi_{n+1}\right)}, \\
\frac{\partial \varphi_{n+1}}{\partial V_{n}}=\frac{-\pi\left(\varphi_{n+1}-\varphi_{n}\right)}{V_{n}-\left(\varphi_{n+1}-\varphi_{n}\right) / \pi+K \sin \left(\varphi_{n+1}\right)}, \\
\frac{\partial V_{n+1}}{\partial \varphi_{n}}=\frac{\partial \varphi_{n+1}}{\partial \varphi_{n}}\left[1 / \pi-2 K \cos \left(\varphi_{n+1}\right)\right]-1 / \pi, \\
\frac{\partial V_{n+1}}{\partial V_{n}}=\frac{\partial \varphi_{n+1}}{\partial V_{n}}\left[1 / \pi-2 K \cos \left(\varphi_{n+1}\right)\right]-1 .
\end{gathered}
$$

Therefore, the Jacobian determinant is given by

$$
\operatorname{det}(J)=\frac{V_{n}+K \sin \left(\varphi_{n}\right)}{V_{n+1}+K \sin \left(\varphi_{n+1}\right)} .
$$

As can be seen in (7) the Jacobian has no constant value once it depends on the dynamical variables associated with the iterations. As a consequence, the system cannot be considered dissipative neither non-dissipative, since $J$ can be greater or less than the unity for distinct regions of the phase space. The phase space volume 
will contract around some regions and expand around some others. This result is important for our purpose of searching small accelerating structures in the phase space, represented by high period AM.

\subsection{Simplified Model}

We define the simplified version considering the position of the platform as fixed, but there is an exchange of momentum and energy between the particle and the moving platform as if the platform were moving. Assuming this approximation, the time elapsed between consecutive impacts can be easily found depending only on the launch velocity of the last collision. So, considering $\left(\varphi_{n+1}-\varphi_{n}\right)=2 \pi V_{n}$, the simplified version of the bouncer model is written as

$$
\left\{\begin{array}{l}
\varphi_{n+1}=\varphi_{n}+2 \pi V_{n} \\
V_{n+1}=\left|V_{n}-2 K \sin \varphi_{n+1}\right|
\end{array} .\right.
$$

The simplified bouncer model defined by (8) is symplectic, as one can easily check. Although the simplified model does not correspond to the full dynamics of a ball bouncing in a moving floor, it can be useful to evaluate analytical calculations about the position and stability of the fixed points. Also, we use the simplified model to visualize the accelerating structures in the modulated phase space, once they will appear as periodic islands due to the symplectic character of this mapping.

\section{Accelerator Modes}

In the phase space, the dynamics of an AM consists of regular and repetitive jumps in $V$ direction. This implies in a ballistic acceleration of the particle without chaotic behaviour. The simplest case happens when the map iteration leads one point in phase space to another at same value of $\varphi$ and shifted in $V$ direction by adding an integer $l$. We designate this kind of dynamics as period-1 AM with step-size $l$. If we impose an artificial periodicity along $V$ direction by modulating the phase space, the period-1 $\mathrm{AM}$ is indistinguishable from a period-1 fixed point. The period of the AM refers to the number of map iterations until a repetition of coordinates in a modulated phase space.

\subsection{Stability of the Period-1 Accelerator Modes Step-Size l}

In this subsection we use the simplified mapping approach to obtain the position of the accelerating structure of period- 1 and step-size $l$ in the phase space as function of the 
control parameter $K$ and estimate their stability. Using the symplectic map described by (8) we obtain the relation for the AM coordinates $V_{n+1}-V_{n}=l=-2 K \sin \left(\varphi_{n+1}\right)$. The position of the period-1 AM step-size $l$ is provided by the mapping in (8) as

$$
\begin{gathered}
V^{*}=l, \\
\varphi^{*}=\arcsin (-l / 2 K) .
\end{gathered}
$$

In order to determine the stability of the period-1 AM step-size $l$, we linearize the system around the position $\left(\varphi^{*}, V^{*}\right)$ by calculating the Jacobian matrix at this point. The calculation of the eigenvalues leads to a characteristic expression as follows

$$
\begin{gathered}
P(\lambda)=\operatorname{det}\left(\begin{array}{cc}
1-\lambda & 2 \pi \\
-2 K \cos \varphi^{*} & 1-4 \pi K \cos \varphi^{*}-\lambda
\end{array}\right), \\
P(\lambda)=\lambda^{2}-\lambda\left(2-4 \pi K \cos \varphi^{*}\right)+1=0 .
\end{gathered}
$$

Since the eigenvalues are complex, the coordinate $\left(\varphi^{*}, V^{*}\right)$ corresponds to an elliptical fixed point, which satisfy the stability condition of the AM. So, we obtain

$$
\left|2-4 \pi K \cos \varphi^{*}\right|<4 \rightarrow 0<4 \pi K \cos \varphi^{*}<4
$$

Replacing the (10) on the expression given by (12) we can express the stability condition of the period-1 AM step-size $l$ as a function of parameter $K$

$$
\sqrt{l^{2} / 4}<K<\sqrt{l^{2} / 4+1 / \pi^{2}} \text {. }
$$

Although the interval of stability of period-1 AM step-size $l$ was calculated by the simplified version model, the results can be extended to the complete bouncer model, where the match of their positions has a good agreement. The obtainment of analytical expressions for high order AM are extremely complicated, even using the simplified model. So, we perform a numerical approach to determine their existence, and the estimation of their stability interval and position in the phase space, as will be explained better in the next subsection.

\subsection{Searching for Accelerator Modes of High Period}

To determine the existence of the high period AM, we obtain numerically the average velocity of a great number of random initial condition merged in the chaotic sea during long time iterations (where each iteration corresponds to one impact) of the complete dynamics for different values of $K$. This procedure is efficient due to the non-symplectic character of the mapping. As a consequence, the map does not 
preserve the area in phase space. So, even that the location of the random initial conditions could be different from the location of accelerating structure, eventually some of them can be "attracted" to the AM [49]. If an AM exists in the non-symplectic mapping, it affects globally the dynamics of the system and the average velocity of the total ensemble will present anomalous diffusion [49].

To perform this analysis, we define the average velocity as

$$
\langle V\rangle_{N}=\frac{1}{M} \sum_{j=1}^{M} \overline{V_{j}}(N, K),
$$

where $M$ represents an ensemble of random initial conditions merged in the chaotic sea, and $\overline{V_{j}}(N, K)$ is expressed by

$$
\overline{V_{j}}(N, K)=\frac{1}{N} \sum_{i=1}^{N} V_{i}
$$

with $N$ representing the total iteration (impact) number,

Although the dynamics is globally affected by the existence of the AM, the high period ones, present low influence on the initial conditions far from their location. To reveal the existence of these high period AM, we set up two distinct averages over the dynamics. First the average was considered for an ensemble of $M=5 \times 10^{4}$, over a time $N=5 \times 10^{3}$ for (14). Then, we also perform the average over a sub-ensemble of $m=500$ initial conditions selected in $M$ that exhibit the highest values of the quantity expressed by (15).

The result obtained by this procedure is shown in Fig. 2, where the black line corresponds to the average over the first ensemble $\left(M=5 \times 10^{4}\right)$ and the red (gray) line corresponds to the average over the sub-ensemble $(m=500)$ most accelerated initial condition. The parameter $K$ was varied with step equal to $\Delta K=0.00005$ between $K=0.13$ (parameter value for which the last spanning curve in phase space is destroyed $[22,49]$ ) and $K=0.5$ (parameter value for which occurs the first period-1 AM [49]).

As we can observe, at some values of the parameter $K$, the quantity $<V>_{5 \times 10^{3}}$ presents peaks very distinguishable from the general behavior of the curves. These peaks indicates the existence of the $\mathrm{AM}$ for the correspondent values of parameter $K$. The computation of the average over the sub-ensemble $m=500$ of the most accelerated initial condition (red (gray) curve) highlights the effect of the AM with less influence in the global dynamics. In most of cases their existence is only revealed by the search of the $m=500$ most accelerated initial condition. The period of each AM obtained by this procedure was numerically determined and indicated over each correspondent peak of Fig. 2. Moreover, the complete information about the AM is organized in Table 1 detailed by the period, range of stability, step-size, parameter of maximum acceleration and position of one accelerating structure. 


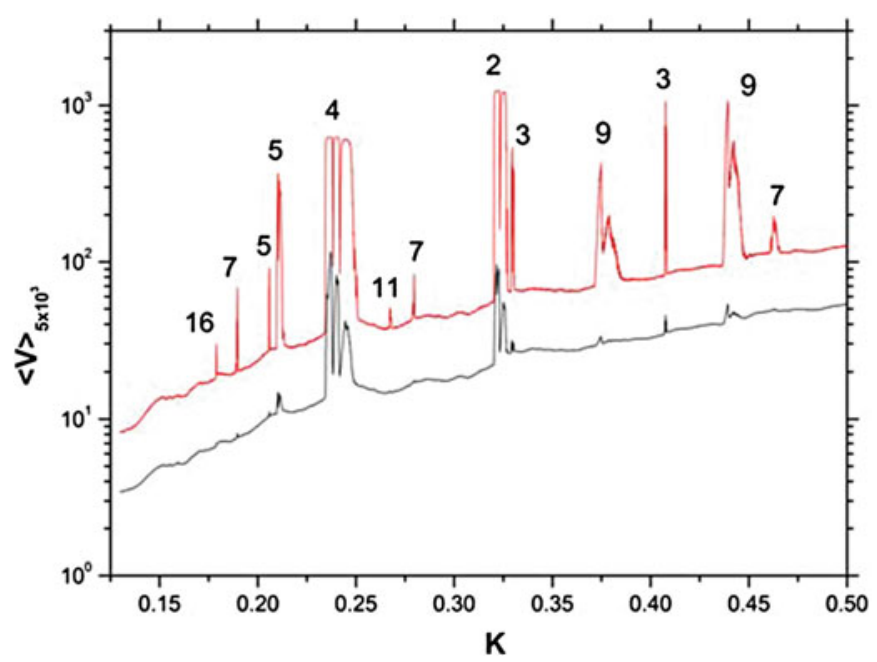

Fig. 2 Average velocity for the Bouncer model as function of $K$. In black we have the average over the first ensemble $M$, and in red (gray) we have the average over the sub-ensemble $m$. For both averages the time series we set at $N=5 \times 10^{3}$. The distinct peaks in both curves indicate the existence of AM and the numbers indicate the correspondent period of the AM (color online)

\section{Islands of Acceleration}

After using the complete model to determine the existence of the AM with less influence in global dynamics, we can use the simplified model to reveal the location, shape and size of the correspondent AM of the complete version. As we discussed, the simplified mapping is symplectic and area-preserving. So, the existence of the AM in this version of the model does not affect the global dynamics of the system but only the initial conditions given inside the AM or very close to them due the effect of stickiness.

When we impose a modulation in $V$ direction of phase space, the AM appear as periodic islands. The number of islands in modulated phase space must be equal to the period of the AM. In many cases, the size of these stability islands is too small to be visualized in the entire modulated phase space. Also, their sizes are strongly related to the influence of the AM in the global dynamics.

In Fig. 3 we illustrate the modulated phase spaces of the simplified mapping with coordinate $\varphi$ at the horizontal direction and $V$ at the vertical direction for the parameters $K^{*}$ of Table 1 . The red (black) circles indicate the position of each accelerating structure (islands in simplified map). As we can see, the number of accelerating structures corresponds to the period of the AM. We can also observe that most of the AM present at least one accelerating structure at $V=1$, with exception of Fig. 3a, e, h. 
Table 1 Accelerator modes for $K<0.5$

\begin{tabular}{|c|c|c|c|c|}
\hline Period & Range of stability & Step-size $(l)$ & $K^{* a}$ & $\begin{array}{l}\text { Accelerating } \\
\text { structure }(\varphi, V) \\
\text { for } K=K^{*}\end{array}$ \\
\hline 16 & $\begin{array}{l}0.17875<K< \\
0.17910\end{array}$ & 1 & 0.17895 & $(-1.847,0.938)$ \\
\hline 7 & $\begin{array}{l}0.18935<K< \\
0.19000\end{array}$ & 1 & 0.18970 & $(-2.431,1.000)$ \\
\hline 5 & $\begin{array}{l}0.20570<K< \\
0.20605\end{array}$ & 1 & 0.20600 & $(-2.520,0.9982)$ \\
\hline 5 & $\begin{array}{l}0.20965<K< \\
0.21325\end{array}$ & 1 & 0.21025 & $(-2.483,1.000)$ \\
\hline 4 & $\begin{array}{l}0.23440<K< \\
0.25065\end{array}$ & 1 & 0.23600 & $(-1.083,0.815)$ \\
\hline 11 & $\begin{array}{l}0.26705<K< \\
0.26810\end{array}$ & 1 & 0.26745 & $(-0.714,1.000)$ \\
\hline 7 & $\begin{array}{l}0.27845<K< \\
0.27980\end{array}$ & 1 & 0.27960 & $(0.865,1.000)$ \\
\hline 2 & $\begin{array}{l}0.32020<K< \\
0.32720\end{array}$ & 1 & 0.32100 & $(-1.182,0.797)$ \\
\hline 3 & $\begin{array}{l}0.32895<K< \\
0.33065\end{array}$ & 1 & 0.32950 & $(-1.357,1.000)$ \\
\hline 9 & $\begin{array}{l}0.37125<K< \\
0.38610\end{array}$ & 4 & 0.37460 & $(-0.943,1.000)$ \\
\hline 3 & $\begin{array}{l}0.40725<K< \\
0.40815\end{array}$ & 2 & 0.40750 & $(-1.398,1.000)$ \\
\hline 9 & $\begin{array}{l}0.43675<K< \\
0.44810\end{array}$ & 6 & 0.43920 & $(-1.046,1.000)$ \\
\hline 7 & $\begin{array}{l}0.46090<K< \\
0.46580\end{array}$ & 2 & 0.46270 & $(-1.088,1.000)$ \\
\hline
\end{tabular}

${ }^{a}$ Parameter value correspondent to the maximum acceleration in the range of stability

In Fig. $4 \mathrm{a}-\mathrm{n}$ we zoomed the modulated phase spaces of Fig. $3 \mathrm{a}-\mathrm{n}$ around one of the accelerating structures, respectively. We consider the modulation $V \bmod [2]$ for a better view of the accelerating structure at $V=1$. The points in red indicate the initial conditions belonging to the AM. We can observe in Fig. 4 that the most of the AM has a reduced area. This is the reason for being so difficult their detection. Also, in the Fig. $4 \mathrm{j}$ and 1 . we reveal that for the period-9 AM, we have three clusters of three accelerating structures on different locations of phase space. This fact increases the effect of stickiness around the islands of acceleration and make their influence more important to study diffusion process, besides the small size of their area on phase space.

The area of accelerating structures is not be the only aspect to determine their influence on the global dynamics in the complete model. As an example, we can compare the Fig. 4c with Fig. 4a, b. The period-5 AM shown in Fig. 4c is the smallest 


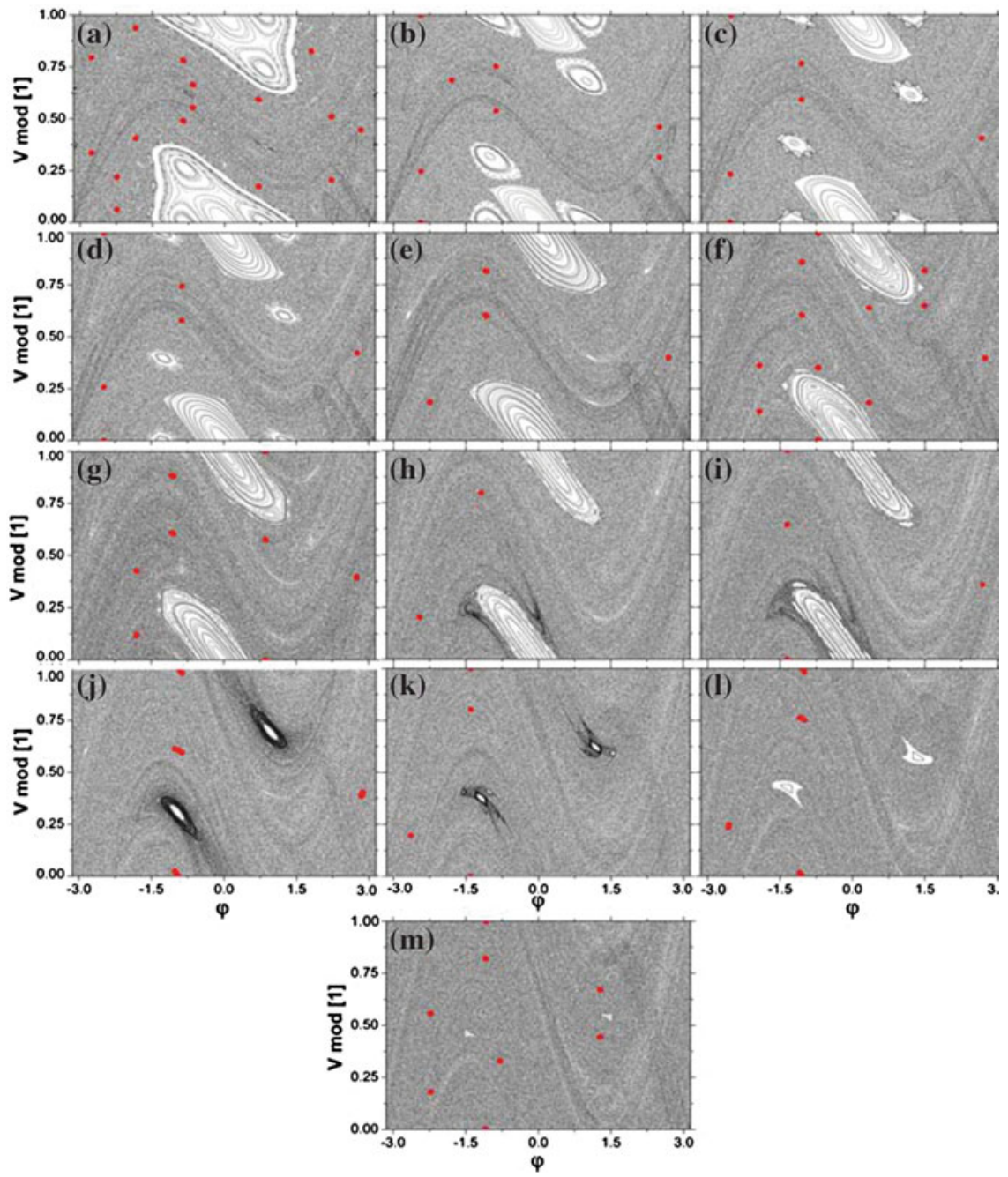

Fig. 3 Modulated phase space for the simplified model with coordinate $\varphi$ on the horizontal direction and $V \bmod [1]$ on the vertical direction for the respective parameters: $\mathbf{a} K=0.17895$, b $K=0.18970$, c $K=0.20600$, d $K=0.21025$, e $K=0.23600$, f $K=0.26745$, g $K=0.27960$, h $K=0.32100$, i $K=0.32950, \mathbf{j} K=0.37460, \mathbf{l} K=0.40750, \mathbf{m} K=0.43920, \mathbf{n} K=0.46270$. The red (black) circles indicate the positions of the accelerating structures (color online)

accelerating structures in direct comparison with the others. However, it is more influent to a global effect in the dynamics than the period-16 AM and period-7 AM as can be seen in Fig. 2. 

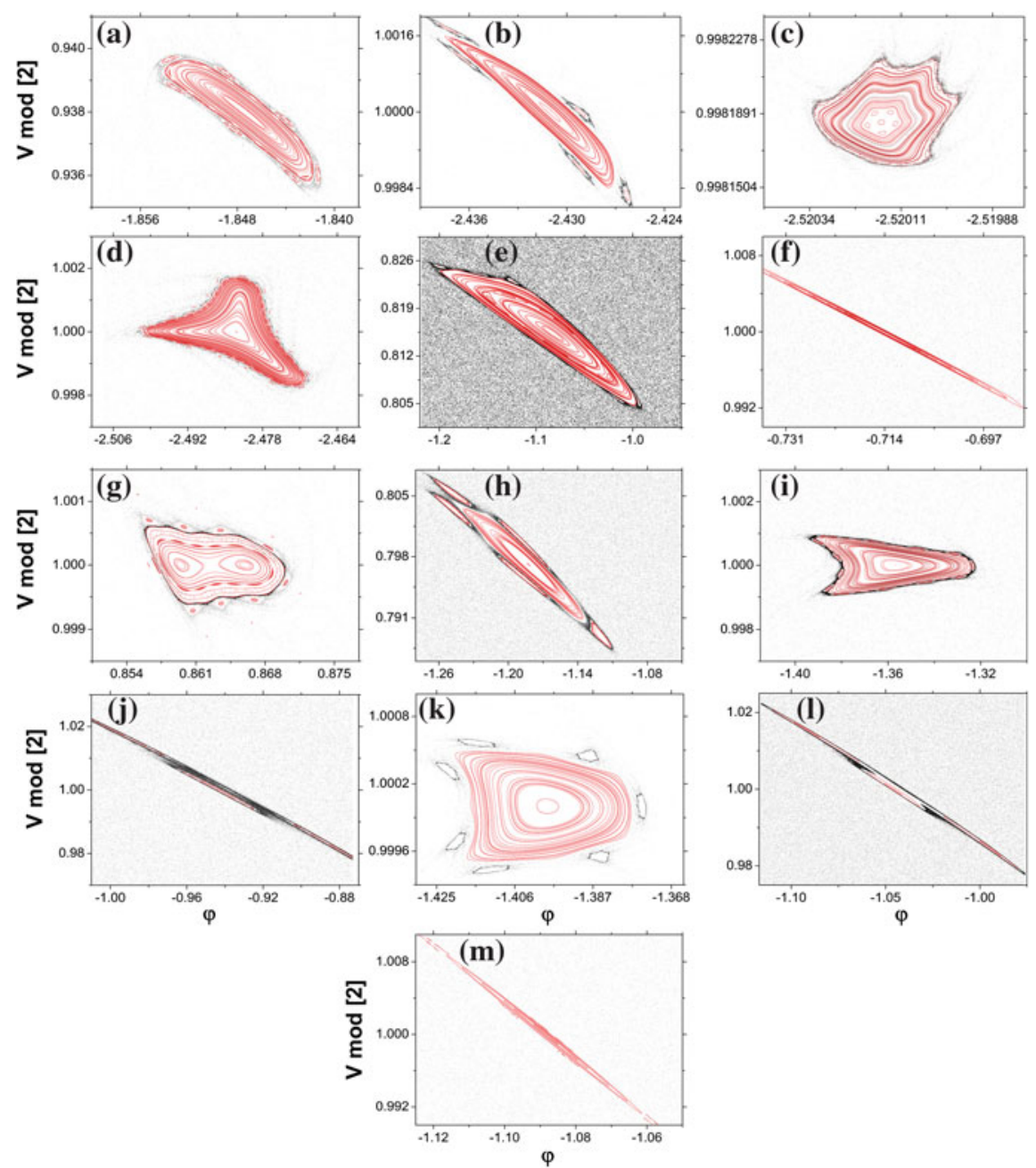

Fig. 4 Islands of Acceleration of the AM presented in Table 1 for the parameters: a $K=0.17895$, b $K=0.18970$, c $K=0.20600$, d $K=0.21025$, e $K=0.23600$, f $K=0.26745$, g $K=0.27960$, h $K=0.32100$, i $K=0.32950, \mathbf{j} K=0.37460, \mathbf{l} K=0.40750$, m $K=0.43920$, n $K=0.46270$

\section{Conclusions}

The existence of high period AM and low influence on the global dynamics was investigated using the Bouncer Model. Due to the global properties of the AM in the complete version of this system, it was used to investigate the distribution of the AM for different periods as we ranged the control parameter. Until now it was a challenging task to detect the existence and location of accelerating structures in symplectic maps, since the initial conditions tested are rarely located inside the 
islands of acceleration (which location is unpredictable in most of the cases). With a model that exhibits a global behaviour for the AM, any sufficiently great ensemble of initial conditions could be used to identify the existence of the AM and their periods.

A simplified version of the model allows us to find a correspondent symplectic mapping similar to the standard mapping. The simplified mapping was used to visualize the accelerating structures and their positions in the phase space. When we impose a modulation between 0 and 1 on the velocity variable, these structures constitute a number of small islands of acceleration equal to the period of the AM. A close view on the accelerating structures reveals that their size are not the only aspect to determine their influence on the global dynamics of the complete model. Other dynamical properties as: period of the AM, step-size $l$ of the jumps, stickiness around accelerating structures, distribution of regular periodic islands in phase space and the chaotic saddle formed by the invariant stable and unstable manifolds should also influence the effect of the AM on the global dynamics of the system [49].

Acknowledgments ALPL acknowledges FAPESP and CNPq for financial support. ILC thanks FAPESP (2011/19296-1) and EDL thanks FAPESP (2012/23688-5), CNPq and CAPES, Brazilian agencies.

\section{References}

1. A.J. Lichtenberg, M.A. Lieberman, Regular and Chaotic Dynamics. Applied Mathematical Sciences, vol 38 (Springer, New York, 1992)

2. R.C. Hilborn, Chaos and Nonlinear Dynamics: An Introduction for Scientists and Engineers (Oxford University Press, New York, 1994)

3. K.T. Alligood, T.D. Sauer, J.A. Yorke, Chaos: An Introduction to Dynamical Systems (Springer, New York, 1996)

4. G.M. Zaslasvsky, Physics of Chaos in Hamiltonian Systens (Imperial College Press, New York, 2007)

5. G.M. Zaslasvsky, Hamiltonian Chaos and Fractional Dynamics (Oxford University Press, New York, 2008)

6. E. Fermi, Phys. Rev. 75, 1169 (1949)

7. M.A. Lieberman, V.A. Godyak, Ieee Trans. Plasma Sci. 26, 955 (1998)

8. A.V. Milovanov, L.M. Zelenyi, Phys. Rev. E 64, 052101 (2001)

9. A. Veltri, V Carbone, Phys. Rev. Lett. 92, 143901 (2004)

10. K. Kobayakawa, Y.S. Honda, T. Samura, Phys. Rev. D 66, 083004 (2002)

11. G. Lanzano et al., Phys. Rev. Lett. 83, 4518 (1999)

12. F. Saif, I. Bialynicki-Birula, M. Fortunato, W.P. Schleich, Phys. Rev. A 58, 4779 (1998)

13. A. Loskutov, A.B. Ryabov, L.G. Akinshin, J. Exp. Theor. Phys. 89, 966 (1999)

14. A. Loskutov, A.B. Ryabov, L.G. Akinshin, J. Phys. A 33, 7973 (2000)

15. R.E. de Carvalho, F.C. Souza, E.D. Leonel, Phys. Rev. E 73, 066229 (2006)

16. F. Lenz, F.K. Diakonos, P. Schmelcher, Phys. Rev. Lett. 100, 014103 (2008)

17. E.D. Leonel, D.F.M. de Oliveira, A. Loskutov, Chaos 19, 033142 (2009)

18. A.L.P. Livorati, A. Loskutov, E.D. Leonel, Physica A 391, 4756 (2012)

19. V. Gelfreich, V. Rom-Kedar, K. Shah, D. Turaev, Phys. Rev. Lett. 106, 074101 (2011)

20. V. Gelfreich, V. Rom-Kedar, D. Turaev, Chaos 22, 033116 (2012)

21. K. Shah, D. Turaev, V. Rom-Kedar, Phys. Rev. E 81, 056205 (2010) 
22. A.L.P. Livorati, T. Kroetz, C.P. Dettmann, I.L. Caldas, E.D. Leonel, Phys. Rev. E 86, 036203 (2012)

23. P.J. Holmes, J. Sound Vibr 84, 173 (1982)

24. L.D. Pustilnikov, Theor. Math. Phys. 57, 1035 (1983)

25. R.M. Everson, Physica D 19, 355 (1986)

26. D. Sternad, M. Duarte, H. Katsumata, S. Schaal, Phys. Rev. E 63, 011902 (2000)

27. N.A. Burnham, A.J. Kulik, G. Gremaud, G.A.D. Briggs, Phys. Rev. Lett. 74, 5092 (1995)

28. A.C.J. Luo, R.P.S. Han, Nonl. Dyn. 10, 1 (1996)

29. J.J. Barroso, M.V. Carneiro, E.E.N. Macau, Phys. Rev. E 79, 026206 (2009)

30. L. Mátyás, R. Klanges, Physica D 187, 165 (2004)

31. E.D. Leonel, A.L.P. Livorati, Commun. Nonl. Sci. Num. Simul. 20, 159 (2015)

32. T.L. Vincent, A.L. Mess, Int. J. Bif. Chaos 10, 579 (2000)

33. S.K. Joseph, I.P. Mariño, M.A.F. Sanjuán, Commun. Nonl. Sci. Num. Simul. 17, 3279 (2012)

34. F. Spahn, U. Schwarz, J. Kurths, Phys. Rev. Lett. 78, 1596 (1997)

35. P. Müller, M. Heckel, A. Sack, T. Pöschel, Phys. Rev. Lett. 110, 254301 (2013)

36. F. Pacheco-Vázquez, F. Ludwig, S. Dorbolo, Phys. Rev. Lett. 113, 1108001 (2014)

37. A.J. Lichtenberg, M.A. Liberman, N.W. Murray, Physica D 28, 371 (1987)

38. A.J. Lichtenberg, M.A. Liberman, Physica D 33, 211 (1988)

39. Y.H. Ichikawa, Y. Nomura, T. Kamimura, Prog. Theor. Phys. Supp. 99, 220 (1989)

40. Y.H. Ichikawa, T. Kamimura, T. Hatori, S.Y. Kim, Prog. Theor. Phys. Supp. 98, 01 (1989)

41. G.M. Zaslavsky, B.A. Niyazov, Phys. Rep. 283, 73 (1997)

42. V. Rom-Kedar, G.M. Zaslavsky, Chaos 9, 697 (1999)

43. S.T. Dembinski, P. Peplowski, Phys. Rev. E 55, 212 (1997)

44. T. Manos, M. Robnik, Phys. Rev. E 87, 062905 (2013)

45. T. Manos, M. Robnik, Phys. Rev. E 89, 022905 (2014)

46. M.K. Oberthaler, R.M. Godun, M.B. d'Arcy, G.S. Summy, K. Burnett, Phys. Rev. Lett. 83, 4447 (1999)

47. S. Schlunk, M.B. d'Arcy, S.A. Gardiner, G.S. Summy, Phys. Rev. Lett. 90, 124102 (2003)

48. S. Fishman, I. Guarneri, L. Rebuzzini, J. Stat. Phys. 110, 911 (2003)

49. T. Kroetz, A.L.P. Livorati, E.D. Leonel, I.L. Caldas, Phys. Rev. E. 92, 012905 (2015) 